\title{
A Complexity-Reduced ML Parametric Signal Reconstruction Method
}

\author{
Z. Deprem, ${ }^{1}$ K. Leblebicioglu, ${ }^{2}$ O. Arıkan, ${ }^{1}$ and A. E. Çetin ${ }^{1}$ \\ ${ }^{1}$ Department of Electrical and Electronics Engineering, Bilkent University, Bilkent, Ankara, 06800, Turkey \\ ${ }^{2}$ Department of Electrical and Electronics Engineering, Middle East Technical University, Ankara, 06531, Turkey
}

Correspondence should be addressed to Z. Deprem, zdeprem@ee.bilkent.edu.tr

Received 2 September 2010; Revised 8 December 2010; Accepted 24 January 2011

Academic Editor: Athanasios Rontogiannis

Copyright ( 2011 Z. Deprem et al. This is an open access article distributed under the Creative Commons Attribution License, which permits unrestricted use, distribution, and reproduction in any medium, provided the original work is properly cited.

\begin{abstract}
The problem of component estimation from a multicomponent signal in additive white Gaussian noise is considered. A parametric ML approach, where all components are represented as a multiplication of a polynomial amplitude and polynomial phase term, is used. The formulated optimization problem is solved via nonlinear iterative techniques and the amplitude and phase parameters for all components are reconstructed. The initial amplitude and the phase parameters are obtained via time-frequency techniques. An alternative method, which iterates amplitude and phase parameters separately, is proposed. The proposed method reduces the computational complexity and convergence time significantly. Furthermore, by using the proposed method together with Expectation Maximization (EM) approach, better reconstruction error level is obtained at low SNR. Though the proposed method reduces the computations significantly, it does not guarantee global optimum. As is known, these types of non-linear optimization algorithms converge to local minimum and do not guarantee global optimum. The global optimum is initialization dependent.
\end{abstract}

\section{Introduction}

In many practical signal applications involving amplitude and/or phase-modulated carrier signals, we encounter discrete-time signals which can be represented as

$$
s[n]=a[n] e^{j \varnothing[n]},
$$

where $a[n]$ and $\varnothing[n]$ are the real amplitude and phase functions, respectively. Such signals are common in radar, sonar applications, and in many other natural problems. A multicomponent [1] signal is a linear combination of these types of signals and is given by

$$
s[n]=\sum_{i=1}^{L} a_{i}[n] e^{j \varnothing_{i}[n]},
$$

where $s_{i}[n]=a_{i}[n] e^{j \varnothing_{i}[n]}$ is the $i$ th component and $L$ is the number of components. Clearly, the linear decomposition of the multicomponent signal in terms of such components is not unique. Some other restrictions should be put on the components to have a unique decomposition [1]. In general, a component is the part of the multicomponent signal which is identifiable in time, in frequency, or in mixed time-frequency plane. Therefore, we will assume that the different components are well separated in time-frequency plane and have a small instantaneous bandwidth compared to separation between components.

The main problem is to separate the components from each other or to recover one of the components. In general the approaches for the solution are those which use nonparametric time-frequency methods and those of parametric ones. In case where the desired signal component is separable or disjoint in one of time or frequency domain, then, with some sort of time or frequency masking, the component can be estimated. When the signals are disjoint either in time or in frequency domain, then time-frequency processing methods are needed for component separation. But, in some cases even though the components are not separated in time or in frequency, the Fractional Fourier Transform [2-4] can be used to separate the components at the fraction, where they are disjoint.

Time Frequency Distribution- (TFD-) based waveform reconstruction techniques, for example, the one in [5], 
synthesize a time-domain signal from its bilinear TFD. In these algorithms, a time-domain signal whose distribution is close to a valid TFD, in a least-squares sense, is searched for.

The well-known time-frequency method is the WignerDistribution [6] based signal synthesis [5, 7-9]. The main drawback related to time-frequency methods is the crossterms and resolution of the time-frequency representations [10]. Therefore, there have been many efforts to obtain crossterm-free and high-resolution TFDs [11-13].

In parametric model a signal or component is represented as a linear combination of some known basis functions $[14,15]$, and the component parameters are estimated. In many radar and sonar applications the polynomials are good basis functions.

If the phase and amplitude functions in (1) are polynomials and amplitude function is constant or slowly varying, the Polynomial Phase Transform (PPT) $[14,16]$ is a practical tool for parameter estimation. While the method is practical, it has difficulties in time-varying amplitude and multicomponent cases [17]. It is also suboptimal since the components are extracted in a sequential manner.

Another solution is the ML estimation of the parameters. The related method is explained in $[15,17]$. The ML estimation of the parameters requires a multivariable nonlinear optimization problem to be solved. Therefore, the solution requires iterative techniques like nonlinear conjugate gradient (NL-CG) or quasi-Newton-type algorithms and is computationally intensive $[15,17]$. Another requirement is a good initial estimate which avoids possible local minima. But it estimates all parameters as a whole and is optimal in this respect. Also it does not suffer from cross-terms related to time-frequency techniques.

In [14] an algorithm is explained which extracts the components using PPT in a sequential manner. In [18] a mixed time-frequency and PPT-based algorithm is proposed. The examples with the ML approach are given in $[15,17]$.

In this paper a method is proposed which uses ML estimation. Similar to [18], the initial estimates are obtained from time-frequency representation of the multicomponent signal and then all parameters are estimated by ML estimation. Since ML estimation requires large amount of computation, a method is proposed to reduce the computations. The proposed method iterates amplitude and phase parameters separately by assuming that the other is known. The method is different from the ones given in $[15,17]$, where the amplitude parameters are eliminated analytically and the resultant equivalent cost function is minimized.

Eliminating amplitude parameters analytically results in a cost function which has less number of parameters. But it is computationally more complex in terms of function and gradient evaluations, which are needed in nonlinear optimization iterations.

With the proposed method, since the cost functions for separate amplitude and phase parameters are less complex, the amount of computation is reduced compared to case where amplitude parameters are eliminated analytically. Furthermore, by using the proposed method in an expectation maximization loop, a better reconstruction error level is obtained. The results are verified with simulations.
In Section 2 we describe the notation and give the explanation of the ML estimation approach which is given in [15]. In Section 3 we describe the proposed method. In Section 4 we compare the computational cost of the proposed method with the case where amplitude parameters are eliminated analytically. In Section 5 we give a brief explanation of Expectation Maximization (EM) and how to use the proposed alternating phase and amplitude minimization method in an EM loop. In Section 6 we drive the CramerRao Bounds on mean square error related to component reconstruction. In Section 7 we present the simulation results. First taking Cramer-Rao bounds as the reference we compare the proposed method with the one given in [15] in terms of mean square reconstruction error and then compare their performance in terms of computational cost.

\section{Problem Formulation and ML Estimation}

Let $x[n]$ be a discrete-time process consisting of the sum of a deterministic multicomponent signal and additive white Gaussian noise given by

$$
x[n]=\sum_{i=1}^{L} a_{i}[n] e^{j \varnothing_{i}[n]}+w[n], \quad n=0,1, \ldots, N-1,
$$

where $w[n]$ is the complex noise process. Denoting $g_{k}[n]$ and $p_{k}[n]$ as the real-valued basis functions for amplitude and phase terms, respectively, we will have

$$
\begin{gathered}
a_{i}[n]=\sum_{k=0}^{P_{i}} a_{i, k} g_{k}[n], \\
\varnothing_{i}[n]=\sum_{k=0}^{Q_{i}} b_{i, k} p_{k}[n],
\end{gathered}
$$

where $a_{i, k}$ and $b_{i, k}$ are the real valued amplitude and phase coefficients for the $i$ th component. Similarly $P_{i}+1$ and $Q_{i}+1$ are the number of coefficients for amplitude and phase functions of the $i$ th component. In general, basis functions can be any functions which are square integrable and spans the space of real and integrable functions in a given observation interval. Also they can be selected to be different for amplitude and phase and for each component. In this paper they are assumed to be polynomial for both amplitude and phase and for all components. Therefore, $P_{i}$ and $Q_{i}$ corresponds to orders for amplitude and phase polynomials of the $i$ th component, respectively.

Defining the amplitude and phase coefficients of the $i$ th component by the vectors

$$
\begin{aligned}
\mathbf{a}_{i} & =\left[\begin{array}{lllll}
a_{i, 0} & a_{i, 1} & a_{i, 2} & \cdots & a_{i, P_{i}}
\end{array}\right]^{T}, \\
\mathbf{b}_{i} & =\left[\begin{array}{lllll}
b_{i, 0} & b_{i, 1} & b_{i, 2} & \cdots & b_{i, Q_{i}}
\end{array}\right]^{T},
\end{aligned}
$$

we can define parameter vectors for all the components as

$$
\begin{aligned}
& \mathbf{a}=\left[\begin{array}{lllll}
\mathbf{a}_{1}^{T} & \mathbf{a}_{2}^{T} & \mathbf{a}_{3}^{T} & \cdots & \mathbf{a}_{L}^{T}
\end{array}\right]^{T}, \\
& \mathbf{b}=\left[\begin{array}{lllll}
\mathbf{b}_{1}^{T} & \mathbf{b}_{2}^{T} & \mathbf{b}_{3}^{T} & \cdots & \mathbf{b}_{L}^{T}
\end{array}\right]^{T} .
\end{aligned}
$$


We will use the following notation

$$
\mathbf{x}=x[\mathbf{n}]=\left[\begin{array}{lllll}
x[0] & x[1] & x[2] & \cdots & x[N-1]
\end{array}\right]^{T},
$$

where

$$
\begin{aligned}
& \mathbf{n}=[0,1,2, \ldots, N-1]^{T}, \\
& \mathbf{w}=w[\mathbf{n}]=\left[\begin{array}{lllll}
w[0] & w[1] & w[2] & \cdots & w[N-1]
\end{array}\right]^{T}, \\
& e^{j \varnothing_{i}[\mathbf{n}]}=\left[\begin{array}{lllll}
e^{j \varnothing_{i}[0]} & e^{j \varnothing_{i}[1]} & e^{j \varnothing_{i}[3]} & \cdots & e^{j \varnothing_{i}[N-1]}
\end{array}\right]^{T} \text {, }
\end{aligned}
$$

where the bold characters $\mathbf{n}, \mathbf{x}, \mathbf{w}$, and $e^{j \varnothing_{i}[\mathbf{n}]}$ are all $N \times 1$ vectors. With these definitions the following matrices can be defined

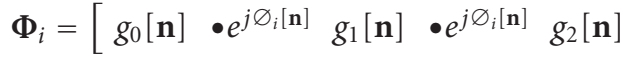

$$
\begin{aligned}
& \left.\bullet e^{j \varnothing_{i}[\mathbf{n}]} \quad \cdots \quad g_{P_{i}}[\mathbf{n}] \cdot e^{j \varnothing_{i}[\mathbf{n}]}\right], \\
& \Phi=\left[\Phi_{1} \Phi_{2} \Phi_{3} \cdots \Phi_{L}\right],
\end{aligned}
$$

where “•” in (10) denotes component-by-component multiplication of vectors. $\boldsymbol{\Phi}_{i}$ s are $N \times\left(P_{i}+1\right)$ matrices which contain the phase parameters only and are defined for each component. The matrix $\Phi$ is an $N \times \sum_{i=1}^{L}\left(P_{i}+1\right)$ matrix and again contains the phase parameters for all components. With these definitions the expression in (3) can be written in matrix notation as

$$
\mathbf{x}=\Phi \mathbf{a}+\mathbf{w}
$$

In this equation the amplitude parameter vector a enters the equation in a linear way, while the phase parameter vector $\mathbf{b}$ enters the equation in nonlinear way through $\Phi$.

Now the problem is to estimate combined parameter vector $\boldsymbol{\theta}=\left[\mathbf{b}^{T} \mathbf{a}^{T}\right]^{T}$ given observed data vector $\mathbf{x}=$ $[x[0] x[1] \quad x[2] \cdots x[N-1]]^{T}$. It is assumed that the observed data length $N$ is sufficiently greater than the total number of estimated parameters given by $M=\sum_{i=1}^{L}\left\{\left(P_{i}+\right.\right.$ 1) $\left.+\left(Q_{i}+1\right)\right\}$.

The number of components, since components are assumed to be well separated on TFD, can be estimated from TFD. But here we will assume that $L$ is known. Similarly $P_{i}$ and $Q_{i}$ are assumed to be known. A method to estimate them can be found in $[14,16]$.

With the additive white Gaussian noise assumption, the probability density function ( $\mathrm{pdf}$ ) of data vector $\mathbf{x}$, given the parameter vector $\boldsymbol{\theta}$ and logarithmic likelihood function, is given by

$$
\begin{gathered}
p(\mathbf{x} \mid \boldsymbol{\theta})=\frac{1}{\left(\pi \sigma^{2}\right)^{\mathbf{N}}} \exp \left\{-\frac{1}{\sigma^{2}}\|\mathbf{x}-\boldsymbol{\Phi} \mathbf{a}\|^{2}\right\}, \\
\Lambda=\log p(\mathbf{x} \mid \boldsymbol{\theta})=-N(\ln \pi+2 \ln \sigma)-\frac{1}{\sigma^{2}}\|\mathbf{x}-\boldsymbol{\Phi} \mathbf{a}\|^{2},
\end{gathered}
$$

where $\sigma^{2}$ is the noise variance? Since $\mathbf{x}$ and $\boldsymbol{\Phi}$ are complex, by defining $\overline{\mathbf{x}}=\left[\operatorname{Re}\{\mathbf{x}\}^{\mathrm{T}} \operatorname{Im}\{\mathbf{x}\}^{\mathrm{T}}\right]^{\mathrm{T}}$ and $\boldsymbol{\Psi}=$ $\left[\operatorname{Re}\{\boldsymbol{\Phi}\}^{\mathrm{T}} \operatorname{Im}\{\boldsymbol{\Phi}\}^{\mathrm{T}}\right]^{\mathrm{T}}$, the log-likelihood function can be rewritten in real quantities as

$$
\Lambda=-N(\ln \pi+2 \ln \sigma)-\frac{1}{\sigma^{2}}\|\overline{\mathbf{x}}-\Psi \mathbf{a}\|^{2} .
$$

Maximizing log likelihood in (15) corresponds to minimizing $f(\mathbf{a}, \mathbf{b})=\|\overline{\mathbf{x}}-\boldsymbol{\Psi} \mathbf{a}\|^{2}$. For a given phase vector $\mathbf{b}$, this cost function is quadratic in amplitude vector $\mathbf{a}$. Therefore, amplitude vector a can be solved analytically as

$$
\hat{\mathbf{a}}=\left(\boldsymbol{\Psi}^{T} \boldsymbol{\Psi}\right)^{-1} \boldsymbol{\Psi}^{T} \overline{\mathbf{x}}
$$

Using this separability feature of the parameter set and substituting (16) in (15) the original log-likelihood function can be replaced by

$$
\Lambda=-N(\ln \pi+2 \ln \sigma)-\frac{1}{\sigma^{2}} J(\mathbf{b}),
$$

where

$$
\begin{aligned}
& J(\mathbf{b})=\overline{\mathbf{x}}^{\mathrm{T}} \mathbf{P}_{\boldsymbol{\Psi}}^{\perp} \overline{\mathbf{x}}, \\
& \mathbf{P}_{\boldsymbol{\Psi}}^{\perp}=\mathbf{I}-\mathbf{P}_{\Psi}, \\
& \mathbf{P}_{\Psi}=\boldsymbol{\Psi}\left(\boldsymbol{\Psi}^{T} \boldsymbol{\Psi}\right)^{-1} \boldsymbol{\Psi}^{T} .
\end{aligned}
$$

While the original cost function was a function of $\mathbf{a}$ and $\mathbf{b}$, this new augmented function is a function of $\mathbf{b}$ only. Like the original cost function this new cost function $J(\mathbf{b})$ is also nonlinear in b. Therefore, minimization requires iterative methods like nonlinear conjugate gradient or quasi-Newtontype methods. These iterative methods require also a good initial estimate to avoid possible local minima. In [15] initial estimates are obtained by PPT. After $\mathbf{b}$ is solved iteratively, a is obtained by (16).

\section{Proposed Method for Iterative Solution}

The separability feature of the original cost function in (15) allows us to reduce the number of unknown parameters via analytical method. Since the resultant cost function is just a function of phase parameters, we will call this method Phase-Only (PO) method. Though PO deals with reduced set of parameters, the resultant cost function $J(\mathbf{b})$ is highly nonlinear and more complicated in terms of function and gradient evaluations. This is a disadvantage when the minimization of the reduced cost function is to be obtained via nonlinear iterative methods. Therefore, in this paper, an alternative method is proposed. The method carries out two minimization algorithms in an alternating manner. The method divides the original minimization problem given by (15) into two subminimizations. The idea is to find one parameter set assuming that the other set is known. First assuming that the initial phase estimate $\hat{\mathbf{b}}^{0}$ is known, the cost function

$$
f_{\mathbf{a}}(\mathbf{a})=f\left(\mathbf{a}, \hat{\mathbf{b}}^{0}\right)=\left\|\overline{\mathbf{x}}-\hat{\boldsymbol{\Psi}}^{0} \mathbf{a}\right\|^{2}
$$


is formed and minimized, and a solution $\hat{\mathbf{a}}^{1}$ is obtained, where $\hat{\Psi}^{0}$ is the matrix obtained by initial phase parameter estimate $\hat{\mathbf{b}}^{0}$. Then using this amplitude estimate $\hat{\mathbf{a}}^{1}$ a second cost function

$$
f_{\mathbf{b}}(\mathbf{b})=f\left(\hat{\mathbf{a}}^{1}, \mathbf{b}\right)=\left\|\overline{\mathbf{x}}-\Psi \hat{\mathbf{a}}^{1}\right\|^{2}
$$

is formed and minimized, and a solution $\hat{\mathbf{b}}^{1}$ is found. These two minimizations constitute one cycle of proposed algorithm. By repeating this cycle, taking $\hat{\mathbf{b}}^{1}$ as the new initial phase estimate, the estimates $\hat{\mathbf{a}}^{2}$ and $\hat{\mathbf{b}}^{2}$ are obtained. By repeating the cycles sufficiently many times, the final estimates $\hat{\mathbf{a}}^{*}$ and $\hat{\mathbf{b}}^{*}$ are obtained as shown in

$$
\hat{\mathbf{b}}^{0} \longrightarrow \hat{\mathbf{a}}^{1} \longrightarrow \hat{\mathbf{b}}^{1} \longrightarrow \hat{\mathbf{a}}^{2} \longrightarrow \hat{\mathbf{b}}^{2} \longrightarrow \hat{\mathbf{a}}^{3} \longrightarrow \hat{\mathbf{b}}^{3} \cdots \hat{\mathbf{a}}^{*} \longrightarrow \hat{\mathbf{b}}^{*} .
$$

The cost function for amplitude parameters $f_{\mathbf{a}}(\mathbf{a})$ is quadratic. Therefore, the solution can be obtained either analytically or via conjugate gradient (CG). But the cost function for phase parameters $f_{\mathbf{b}}(\mathbf{b})$ is nonlinear. Therefore, we need to use nonlinear methods.

The proposed method, which we will call, from now on, Alternating Phase and Amplitude (APA) method, is a generalization of the so-called coordinate descent method [19], where the minimization of a multivariable function is done by sequentially minimizing with respect to a single variable or coordinate and keeping the others fixed. By cyclically repeating the same process a minimum for the function is searched. A generalization of coordinate descent method is the Block Coordinate Descent (BCD) method, where the variables are separated into blocks containing more than one variable and the minimization is done over a block of variables and keeping the others fixed. In our case we have two blocks, and the minimization over one block is quadratic. Though the indications on the convergence of similar algorithms are given in [19], the theoretical proof regarding the convergence of proposed method is beyond the scope of this work, and we will content with the simulation results.

The main trick with proposed algorithm is that during amplitude and phase minimizations we do not have to find the actual minimum. What we are looking for is a sufficient improvement from the current estimate that we have. Therefore, for the phase iterations rather than iterating down to the convergence point we can iterate a sufficient number of iterations to get some improvement. The same is valid for the minimization of $f_{\mathbf{a}}(\mathbf{a})$ if we decide to use conjugate gradient. But overall alternating phase and amplitude iterations will allow us to converge to a minimum. The first minimization can be chosen to be the minimization of $f_{\mathbf{b}}(\mathbf{b})$ instead of $f_{\mathbf{a}}(\mathbf{a})$. Then the sequence in (23) will start by $\widehat{\mathbf{a}}^{0}$. The decision about which one to start with should be based on which initial parameter vector, $\hat{\mathbf{a}}^{0}$ or $\hat{\mathbf{b}}^{0}$, is more close to its actual. This cannot be known in advance, but, based on success of the method by which the initial estimates $\hat{\mathbf{a}}^{0}$ and $\widehat{\mathbf{b}}^{0}$ are obtained, a decision can be given.

Like $J(\mathbf{b}), f_{\mathbf{b}}(\mathbf{b})$ is also nonlinear, and we need iterative methods like nonlinear conjugate gradient or quasi-Newton.
These methods converge to local minimum and do not guarantee global minimum unless initial estimates are sufficiently close to global optimum. Therefore, we need to find a method which gives us initial estimates. While in [15] initial estimates are obtained by PPT, in this paper we obtained the initial estimates from time-frequency methods. The time-frequency distribution we used is the Short-Time Fourier Transform (STFT).

At first cycle, the phase iterations will be started by $\hat{\mathbf{b}}^{0}=$ $\hat{\mathbf{b}}^{\mathrm{TF}}$ where $\hat{\mathbf{b}}^{\mathrm{TF}}$ is the estimate obtained from time-frequency method. In later cycles, the previous cycle estimates will be used. If minimization of $f_{\mathbf{a}}(\mathbf{a})$ is done analytically, then we will not need any initial value. But, if we decide to use iterative methods again, we can use initial estimate $\hat{\mathbf{a}}^{0}=\hat{\mathbf{a}}^{\mathrm{TF}}$ obtained from time-frequency method.

As we stated before we assume that the different components are well separated in time-frequency plane and have a small instantaneous bandwidth; that is, the components are not crossing each other. Therefore, by using magnitude STFT, the ridges of each component are detected on TF plane. The algorithm detects the ridges on TF plane by detecting local frequency maximums for each time index. Also by using a threshold the effect of noise is reduced, and the IF is detected at points where component is stronger than noise. Therefore, even though when the weak end of some components is interfering on TF plane with some other stronger component, the IF of stronger component is detected at that point, but the week part of other components is not detected. But the estimates obtained with this method, though they are not the best ones, will be sufficient as initial parameters.

Then from the ridges the instantaneous frequency (IF) samples $\left(\hat{f}_{i}[n]\right)$ for each component are estimated and by polynomial fit corresponding polynomial is obtained. Then by integrating this polynomial the phase function $\widehat{\varnothing}_{i}[n]$ and polynomial coefficients $\hat{\mathbf{b}}_{i}^{\mathrm{TF}}$ for each component are obtained. By dechirping $x[n]$ by $e^{-j \widehat{\varnothing}_{i}[n]}$ and low-pass filtering the result, the amplitude estimate $\hat{a}_{i}[n]$ is obtained for each component. Again by polynomial fit $\hat{\mathbf{a}}_{i}^{\mathrm{TF}}$ is obtained for each component. The overall steps for the proposed APA algorithm are summarized in Table 1.

The initial estimates are obtained from signal TFD by steps 1-5 given in Table 1. Some other methods could also be used. But in this paper the main focus is on the last step. Therefore, though the steps $1-5$ were implemented, the efficiency and performance of this part have not been studied in detail. The only concern was to get initial estimates which are close enough to actual values to avoid local minima if possible. But it should be noted that for the comparison purposes the same initial conditions will be used for the proposed APA algorithm and the phase-only method given in [15].

An important issue that we need to question is the uniqueness of the solution to the optimization problem in (15). Since we express a component in terms of amplitude and phase functions and these functions are expressed in terms of basis functions, we need to question the uniqueness of the global optimum at three levels. 
Starting form last level, given a phase function $\varnothing_{i}[n]$, uniqueness of the parameter vector $\mathbf{b}_{i}$ for this function can be assured if the base functions $p_{k}[n], k=0,1, \ldots, Q_{i}$, are independent of each other. The same is valid for amplitude function $a_{i}[n]$ and parameter vector $\mathbf{a}_{i}$.

Uniqueness at the amplitude and phase function level (model functions level) will not be assured due to phase ambiguity, because if $a_{i}[n]$ and $\varnothing_{i}[n]$ constitute a component then $-a_{i}[n]$ and $\varnothing_{i}[n]+\pi$ will also constitute the same component. Therefore, even though $\mathbf{a}_{i}$ is unique for $a_{i}[n]$ and $\mathbf{b}_{i}$ is unique for $\varnothing_{i}[n]$, the pair $a_{i}[n]$ and $\varnothing_{i}[n]$ will not be unique for $s_{i}[n]$ and, as a result, $\theta_{i}=\left[\mathbf{b}_{i}^{T} \mathbf{a}_{i}^{T}\right]^{T}$ will not be unique for $s_{i}[n]$. This shows that the global optimum is not unique in terms of model functions, hence in terms of parameter vector $\boldsymbol{\theta}=\left[\mathbf{b}^{T} \mathbf{a}^{T}\right]^{T}$.

On the other hand uniqueness at signal $s_{i}[n]$ or component level will be possible if the components are well separated on TFD [1]. In simple terms if no component is coinciding at the same time-frequency point with some other component then the components which constitute the sum in (2) can be found uniquely. Two extreme cases are those where all components are separated in time domain or in frequency domain.

Therefore, even though uniqueness is not satisfied at model functions level hence at parameter level, it can be satisfied at component or signal level with the restrictions on time-frequency plane. In fact, the solution ambiguity in model or parameter space will not affect the final performance of the component reconstruction as long as the combination of model functions or model parameters gives the same signal or component. In our case we extract the initial parameters for a component from related TF area which is disjoint. Therefore, assuming that the initial parameters are close enough to global optimum, we use these restrictions, which will make the component level uniqueness possible, at the beginning.

On the contrary to the assumptions made on time frequency support of components, in simulations, one example (Ex2) is selected such that the components are slightly crossing each other. But most of the parts are nonoverlapping, and these parts allow estimation of an initial IF which will help uniqueness, because, we have assumed in Section 2 that the phase orders $Q_{i}$ s are also known. With this assumption, the set of ambiguous IF estimates hence phase estimates are eliminated for this example, because fitting other ambiguous IFs to the known polynomial order will result in higher fit error. Therefore, for similar examples, the time-frequency restriction can be slightly relaxed.

3.1. Computational Cost Analysis. With the phase-only method the resultant cost function $J(\mathbf{b})$ is given by (18). For the sake of computation ease if we reorganize this equation we will have

$$
J(\mathbf{b})=\overline{\mathbf{x}}^{\mathrm{T}} \mathbf{P}_{\boldsymbol{\Psi}}^{\perp} \overline{\mathbf{x}}=\overline{\mathbf{x}}^{\mathrm{T}} \overline{\mathbf{x}}-\left(\boldsymbol{\Psi}^{T} \overline{\mathbf{x}}\right)^{\mathrm{T}}\left(\boldsymbol{\Psi}^{T} \boldsymbol{\Psi}\right)^{-1} \boldsymbol{\Psi}^{T} \overline{\mathbf{x}},
$$

where $\boldsymbol{\Psi}=\left[\begin{array}{lllll}\boldsymbol{\Psi}_{1} & \boldsymbol{\Psi}_{2} & \boldsymbol{\Psi}_{3} & \ldots & \boldsymbol{\Psi}_{L}\end{array}\right]$ and $\boldsymbol{\Psi}_{i}$ is given by

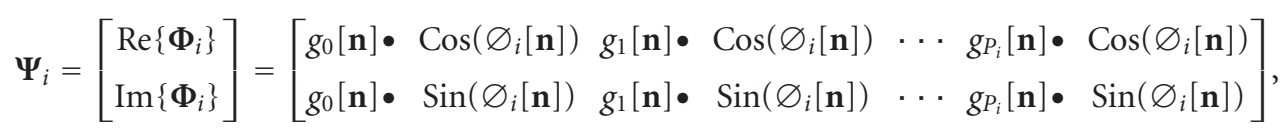

where "•" again denotes component-by-component multiplication of vectors.

The gradient of $J(\mathbf{b})$ is given by [15]

$$
\nabla J(\mathbf{b})=-2 \overline{\mathbf{x}}^{\mathrm{T}} \mathbf{P}_{\Psi}^{\perp} \mathbf{B},
$$

where

$$
\begin{gathered}
\mathbf{B}=\left[\mathbf{B}_{1}, \mathbf{B}_{2}, \ldots, \mathbf{B}_{L}\right], \\
\mathbf{B}_{i}=\left[\widetilde{\mathbf{b}}_{i, 0}, \widetilde{\mathbf{b}}_{i, 1}, \widetilde{\mathbf{b}}_{i, 2}, \ldots, \widetilde{\mathbf{b}}_{i, Q_{i}}\right], \\
\widetilde{\mathbf{b}}_{i, k}=\frac{\partial \Psi_{i}}{\partial b_{i, k}} \mathbf{R}_{i}^{T} \overline{\mathbf{x}} \quad k=0,1, \ldots, Q_{i}, \\
\mathbf{R}=\Psi\left(\boldsymbol{\Psi}^{T} \boldsymbol{\Psi}\right)^{-1}=\left[\mathbf{R}_{1}, \mathbf{R}_{2}, \ldots, \mathbf{R}_{L}\right] .
\end{gathered}
$$

The derivative of $\boldsymbol{\Psi}_{i}$ with respect to $b_{i, k}$ is computed as follows:

$$
\frac{\partial \Psi_{i}}{\partial b_{i, k}}=\tilde{\Psi}_{i} \cdot \mathbf{G}_{k}
$$

where $\tilde{\boldsymbol{\Psi}}_{i}$ is the reordered version of $\boldsymbol{\Psi}_{i}$ given by

$$
\widetilde{\boldsymbol{\Psi}}_{i}=\left[\begin{array}{c}
-\operatorname{Im}\left\{\boldsymbol{\Phi}_{i}\right\} \\
\operatorname{Re}\left\{\boldsymbol{\Phi}_{i}\right\}
\end{array}\right]
$$

and $\mathbf{G}_{k}$ has the same dimensions as $\widetilde{\boldsymbol{\Psi}}_{i}$ and at each column contains the same $2 N \times 1$ vector $\left[\begin{array}{c}p_{k}[\mathbf{n}] \\ p_{k}[\mathbf{n}]\end{array}\right]$. The multiplication between $\tilde{\boldsymbol{\Psi}}_{i}$ and $\mathbf{G}_{k}$ is component by component.

With the proposed method, the minimization of $f_{\mathbf{a}}(\mathbf{a})$ either by CG or analytically is relatively easy. Similarly the computation of $f_{\mathbf{b}}(\mathbf{b})=\left\|\overline{\mathbf{x}}-\Psi \hat{\mathbf{a}}^{0}\right\|^{2}$ is also easy. By defining $\mathbf{z}=\Psi \hat{\mathbf{a}}^{0}=\sum_{i=1}^{L} \mathbf{z}_{i}$

$\mathbf{z}_{i}=\Psi_{i} \hat{\mathbf{a}}_{i}^{\mathbf{0}}=\left[\begin{array}{c}\operatorname{Re}\{\boldsymbol{\Phi}\} \\ \operatorname{Im}\{\boldsymbol{\Phi}\}\end{array}\right] \hat{\mathbf{a}}_{i}^{\mathbf{0}}=\left[\begin{array}{c}\mathbf{z}_{i R} \\ \mathbf{z}_{i I}\end{array}\right]=\left[\begin{array}{l}\sum_{i} \hat{a}_{i, k}^{0} g_{k}[\mathbf{n}] \bullet \operatorname{Cos}\left(\varnothing_{i}[\mathbf{n}]\right) \\ k=0 \\ \sum_{i} \hat{a}_{i, k}^{0} g_{k}[\mathbf{n}] \bullet \operatorname{Sin}\left(\varnothing_{i}[\mathbf{n}]\right)\end{array}\right]$, 
TABLE 1: The proposed alternating phase and amplitude (APA) algorithm.

\begin{tabular}{ll}
\hline 1 & Compute $|\mathrm{STFT}|$ for $x[n]$, and detect the ridges and the number of components $L$ \\
2 & Compute $\hat{f}_{i}[n]$ and $\hat{f}_{i}(t)$ via polynomial fit \\
3 & Compute $\widehat{\varnothing}_{i}(t)=2 \pi \int_{0}^{t} \widehat{f}_{i}(\tau) d \tau+\widehat{\varnothing}_{i}(0)$ and $\widehat{\varnothing}_{i}[n]$ determine $\hat{\mathbf{b}}_{i}^{\mathrm{TF}}$ where $\widehat{\varnothing}_{i}(0)$ is the phase offset estimated from data \\
4 & Compute $x[n] e^{-j \widehat{\varnothing}_{i}[n]}$ and low-pass filter to get $\hat{a}_{i}[n]$ \\
5 & Using polynomial fit get $\hat{\mathbf{a}}_{i}^{\mathrm{TF}}$ \\
6 & Minimize $f_{\mathbf{b}}(\mathbf{b})$ and $f_{\mathbf{a}}(\mathbf{a})$ in an alternating manner using $\hat{\mathbf{a}}^{0}=\hat{\mathbf{a}}^{\mathrm{TF}}$ and $\hat{\mathbf{b}}^{0}=\hat{\mathbf{b}}^{\mathrm{TF}}$ \\
\hline
\end{tabular}

TABLE 2: Minimization of $J(\mathbf{b})$ with quasi-Newton (BFGS) algorithm.

\begin{tabular}{lcr}
\hline & Phase iterations: minimization of $J(\mathbf{b})$ with quasi-Newton $(\mathrm{BFGS})$ algorithm \\
\hline Step & Computation & Multiplication cost \\
\hline Initial & $\mathbf{H}_{0}=\mathbf{I}_{\mathbf{N}_{b}}$ & $N_{\mathbf{b}}^{2}$ \\
1 & $\mathbf{d}_{k}=-\mathbf{H}_{k} \nabla J\left(\mathbf{b}_{(k)}\right)$ & $F_{k}\left\{2 N\left(0.5 N_{\mathbf{a}}^{2}+2.5 N_{\mathbf{a}}+N_{\mathbf{b}}+10 L\right)+N_{\mathbf{a}}^{3}+N_{\mathbf{a}}^{2}+N_{\mathbf{a}}\right\}$ \\
2 & $\alpha_{k}=\min _{\alpha} J\left(\mathbf{b}_{(k)}+\alpha \mathbf{d}_{k}\right)$ & $N_{\mathbf{b}}$ \\
3 & line search with Wolfe Conditions & $G_{k}\left\{2 N\left(1.5 N_{\mathbf{a}}^{2}+3.5 N_{\mathbf{a}}+2 N_{\mathbf{b}}+2 \sum_{i=1}^{L} \bar{P}_{i} \bar{Q}_{i}+10 L+1\right)+N_{\mathbf{a}}^{3}\right\}$ \\
& $\mathbf{b}_{(k+1)}=\mathbf{b}_{(k)}+\alpha \mathbf{d}_{k}$ & $N_{\mathbf{b}}+1$ \\
4 & $\mathbf{s}_{k}=\mathbf{b}_{(k+1)}-\mathbf{b}_{(k)}$ & \\
& $\mathbf{y}_{k}=\nabla J\left(\mathbf{b}_{(k+1)}\right)-\nabla J\left(\mathbf{b}_{(k)}\right)$ & \\
5 & $\rho_{k}=1 /\left(\mathbf{y}_{k}^{T} \mathbf{s}_{k}\right)$ & \\
\hline
\end{tabular}

TABLE 3: Minimization of $f_{\mathbf{b}}(\mathbf{b})$ with quasi-Newton (BFGS) algorithm.

\begin{tabular}{|c|c|c|}
\hline \multicolumn{3}{|c|}{ Phase iterations: minimization of $f_{\mathbf{b}}(\mathbf{b})$ with quasi-Newton (BFGS) Algorithm } \\
\hline Step & Computation & Multiplication cost \\
\hline Initial & $\mathbf{H}_{0}=\mathbf{I}_{\mathbf{N}_{b}}$ & \\
\hline 1 & $\mathbf{d}_{k}=-\mathbf{H}_{k} \nabla f_{\mathbf{b}}\left(\mathbf{b}_{(k)}\right)$ & $N_{\mathbf{b}}^{2}$ \\
\hline \multirow{2}{*}{2} & $\alpha_{k}=\min _{\alpha} f_{\mathbf{b}}\left(\mathbf{b}_{(k)}+\alpha \mathbf{d}_{k}\right)$ & $2 \mathrm{NF}_{k}\left\{N_{\mathbf{a}}+N_{\mathbf{b}}+11 L+1\right\}$ \\
\hline & line search with Wolfe Conditions & $2 \mathrm{NG}_{k}\left\{N_{\mathrm{a}}+3 N_{\mathrm{b}}+11 L+1\right\}$ \\
\hline 3 & $\mathbf{b}_{(k+1)}=\mathbf{b}_{(k)}+\alpha \mathbf{d}_{k}$ & $N_{\mathrm{b}}$ \\
\hline 4 & $\begin{array}{c}\mathbf{s}_{k}=\mathbf{b}_{(k+1)}-\mathbf{b}_{(k)} \\
\mathbf{y}_{k}=\nabla f_{\mathbf{b}}\left(\mathbf{b}_{(k+1)}\right)-\nabla f_{\mathbf{b}}\left(\mathbf{b}_{(k)}\right) \\
\rho_{k}=1 /\left(\mathbf{y}_{k}^{T} \mathbf{s}_{k}\right)\end{array}$ & $N_{\mathbf{b}}+1$ \\
\hline 5 & $\mathbf{H}_{k+1}=\left(\mathbf{I}-\rho_{k} \mathbf{s}_{k} \mathbf{y}_{k}^{T}\right) \mathbf{H}_{k}\left(\mathbf{I}-\rho_{k} \mathbf{y}_{k} \mathbf{s}_{k}^{T}\right)+\rho_{k} \mathbf{s}_{k} \mathbf{s}_{k}^{T}$ & $5 N_{\mathbf{b}}^{2}+3 N_{\mathbf{b}}$ \\
\hline
\end{tabular}

we can rewrite

$$
f_{\mathbf{b}}(\mathbf{b})=\left\|\overline{\mathbf{x}}-\Psi \hat{\mathbf{a}}^{0}\right\|^{2}=\|\overline{\mathbf{x}}-\mathbf{z}\|^{2} .
$$

Using (32)-(34) the gradient of $f_{\mathbf{b}}(\mathbf{b}), \nabla f_{\mathbf{b}}(\mathbf{b})$ is obtained as

$$
\begin{aligned}
& \nabla f_{\mathbf{b}}(\mathbf{b}) \\
& =-2(\overline{\mathbf{x}}-\mathbf{z})^{T} \\
& \times\left[\frac{\partial \mathbf{z}}{\partial b_{1,0}} \frac{\partial \mathbf{z}}{\partial b_{1,1}} \cdots \frac{\partial \mathbf{z}}{\partial b_{1, Q_{1}}} \cdots \frac{\partial \mathbf{z}}{\partial b_{L, 0}} \frac{\partial \mathbf{z}}{\partial b_{L, 1}} \cdots \frac{\partial \mathbf{z}}{\partial b_{L, Q_{L}}}\right]
\end{aligned}
$$

where

$$
\frac{\partial \mathbf{z}}{\partial b_{i, l}}=\left[\begin{array}{c}
-\mathbf{z}_{i I} \\
\mathbf{z}_{i R}
\end{array}\right] \cdot\left[\begin{array}{c}
p_{l}[\mathbf{n}] \\
p_{l}[\mathbf{n}]
\end{array}\right] .
$$

Considering (24)-(29) and (30)-(33) it is apparent that function and gradient evaluations for $J(\mathbf{b})$ are much more complicated compared to $f_{\mathbf{b}}(\mathbf{b})$ and $f_{\mathbf{a}}(\mathbf{a})$. But in order to get a tangible comparison a computational cost analysis has been done and the results are summarized in Tables 2-4, where, the analysis is based on the assumption that both for the minimization of $J(\mathbf{b})$ and $f_{\mathbf{b}}(\mathbf{b})$ the quasi-Newton algorithm BFGS [19] is used. 
TABLE 4: Minimization of $f_{\mathbf{a}}(\mathbf{a})$ with conjugate gradient (CG).

\begin{tabular}{lcc}
\hline \multicolumn{3}{c}{ Amplitude iterations: minimization of $f_{\mathbf{a}}(\mathbf{a})$ with conjugate gradient } \\
\hline Step & Computation & Multiplication cost \\
\hline \multirow{2}{*}{ Initial } & $\mathbf{A}=\boldsymbol{\Psi}^{T} \boldsymbol{\Psi}, \mathbf{y}=\mathbf{\Psi}^{T} \overline{\mathbf{x}}$ & \\
& $\mathbf{r}_{0}=\mathbf{y}-\mathbf{A} \mathbf{a}_{(0)}=\boldsymbol{\Psi}^{T}\left(\overline{\mathbf{x}}-\boldsymbol{\Psi} \mathbf{a}_{(0)}\right)$ & \\
1 & $\mathbf{d}_{0}=\mathbf{r}_{0}$ & \\
2 & $\alpha_{\mathbf{i}}=\left(\mathbf{r}_{i}^{T} \mathbf{r}_{i}\right) /\left(\mathbf{d}_{i}^{T} \mathbf{A d}_{i}\right)$ & $\left.N_{\mathbf{b}}+10 L\right)$ \\
3 & $\mathbf{a}_{(i+1)}=\mathbf{a}_{(i)}+\alpha_{\mathbf{i}} \mathbf{d}_{i}$ & $N_{\mathbf{a}}+1$ \\
4 & $\mathbf{r}_{i+1}=\mathbf{r}_{i}-\alpha_{i} \mathbf{A d}_{i}$ & $N_{\mathbf{a}}$ \\
5 & $\beta_{i+1}=\left(\mathbf{r}_{i+1}^{T} \mathbf{r}_{i+1}\right) /\left(\mathbf{r}_{i}^{T} \mathbf{r}_{i}\right)$ & $N_{\mathbf{a}}$ \\
& $\mathbf{d}_{i+1}=\mathbf{r}_{i+1}+\beta_{i+1} \mathbf{d}_{i}$ & $N_{\mathbf{a}}+1$ \\
\end{tabular}

The second columns in Tables $2-4$ give the required computation for each step during one BFGS or CG iteration. The last columns give the number of multiplications per step. where $\bar{P}_{i}=P_{i}+1$ and $\bar{Q}_{i}=Q_{i}+1$ represent number of parameters for amplitude and phase functions of the $i$ th component. Parameters $N_{\mathrm{a}}=\sum_{i=1}^{L} \bar{P}_{i}$ and $N_{\mathbf{b}}=\sum_{i=1}^{L} \bar{Q}_{i}$ represent total number of amplitude and phase parameters for all components, respectively. $F_{k}$ and $G_{k}$ represent $\mathbf{b}_{(k)}$ denotes phase parameter vector for all the components at $k$ th iteration of BFGS. In order to differentiate it from the $\mathbf{b}_{i}$, which is the phase parameter vector for the $i$ th component, the index is taken into parenthesis. Similarly $\mathbf{a}_{(i)}$ denotes amplitude parameter vector for all the components at $i$ th iteration of conjugate gradient.

During computation cost analysis some assumptions were made. For example, the matrix inversion cost of an $N_{\mathrm{a}} \times N_{\mathrm{a}}$ matrix was taken as $N_{a}^{3}$ multiplications. These types of assumptions do not alter main results but allow us to get a final value.

Considering the phase iterations for $J(\mathbf{b})$ in Table 2 and phase iterations for $f_{\mathbf{b}}(\mathbf{b})$ in Table 3 , we can see that the main step which contributes to the computations is the line search step. This step requires the function and gradient evaluations. Also, comparing the computation cost at this step in parenthesis we see that while for $J(\mathbf{b})$ the computation cost is $O\left(N N_{\mathbf{a}}^{2}\right)+O\left(N N_{\mathbf{b}}\right)+O\left(N \sum_{i=1}^{L} \bar{P}_{i} \bar{Q}_{i}\right)$, it is $O\left(N N_{\mathbf{a}}\right)+$ $O\left(N N_{\mathbf{b}}\right)$ for $f_{\mathbf{b}}(\mathbf{b})$.

If minimization of $f_{\mathbf{a}}(\mathbf{a})$ is done via conjugate gradient (CG) algorithm then the computation cost is given in Table 4. But, if minimum is found analytically, then the cost of (16) need to be taken into account. Using similar calculation analysis it will be found that cost of finding minimum of $f_{\mathbf{a}}(\mathbf{a})$ is approximately $2 N\left(2 N_{a}+N_{\mathbf{b}}+10 L\right)+2 N_{a}^{3}+$ $N_{a}^{2}$.

For a better comparison of APA and PO methods we need to consider overall complexity of two methods. For the minimization of $J(\mathbf{b})$ we need to compute the cost of each BFGS iteration, which consists of 5 steps, and multiply with the number of iterations. On the other hand, for the proposed APA method we need to compute the cost of minimizing $f_{\mathbf{b}}(\mathbf{b})$ and plus the cost of minimizing $f_{\mathbf{a}}(\mathbf{a})$ and multiply the result with the number of cycles of alternating phase and amplitude minimizations.
The cost of line search step in minimization of $J(\mathbf{b})$ and $f_{\mathbf{b}}(\mathbf{b})$ with BFGS requires the number of function and gradient evaluations to be known. But, the actual numbers of the evaluations are not known beforehand. Therefore we need to find them via simulations.

\section{Expectation Maximization with Alternating Phase and Amplitude Method}

In ML estimation the aim is to maximize the conditional pdf $p(\mathbf{x} \mid \boldsymbol{\theta})$ or its logarithm, that is, $L(\boldsymbol{\theta})=\log p(\mathbf{x} \mid \boldsymbol{\theta})$, where, $\mathbf{x}$ is the observation data vector, $\boldsymbol{\theta}$ is the parameter vector to be estimated, and $L(\boldsymbol{\theta})$ is the logarithmic likelihood function. In most of cases, if the pdf is not Gaussian, analytic maximization is difficult. Therefore, the Expectation Maximization (EM) $[20,21]$ procedure is used to simplify the maximization iteratively.

The key idea underlying EM is to introduce a latent or hidden variable $\mathbf{z}$ whose pdf depends on $\boldsymbol{\theta}$ with the property $p(\mathbf{z} \mid \boldsymbol{\theta})$ whose maximizing is easy or, at least, easier than maximizing $p(\mathbf{x} \mid \boldsymbol{\theta})$. The observed data $\mathbf{x}$ without hidden or missing data is called incomplete data.

EM is an efficient iterative procedure to compute the Maximum Likelihood (ML) estimate in the presence of missing or hidden data. In other words, the incomplete data $\mathbf{x}$ is enhanced by guessing some useful additional information. The hidden vector $\mathbf{z}$ is called as complete data in the sense that, if it were fully observed, then estimating $\boldsymbol{\theta}$ would be an easy task.

Technically $\mathbf{z}$ can be any variable such that $\boldsymbol{\theta} \rightarrow \mathbf{z} \rightarrow \mathbf{x}$ is a Markov chain, that is, $\mathbf{z}$ is such that, $p(\mathbf{x} \mid \mathbf{z}, \boldsymbol{\theta})$ is independent of $\boldsymbol{\theta}$. Therefore, we have

$$
p(\mathbf{x} \mid \mathbf{z}, \boldsymbol{\theta})=p(\mathbf{x} \mid \mathbf{z})
$$

While in some problems there are "natural" hidden variables, in most of the cases they are artificially defined.

In ML parameter estimation given in Section 2 the EM method is applied as follows. Assume that we would like to estimate the amplitude and phase parameters $\mathbf{a}_{k}$ and $\mathbf{b}_{k}$ for the $k$ th component given the data $x[n]$ expressed by (3). The data is incomplete in the sense that it includes the linear 
TABLE 5: Expectation Maximization (EM) iteration steps.

\begin{tabular}{ll}
\hline & EM steps for multicomponent signal parameter estimation \\
\hline Step & Operation \\
\hline Initial & Get initial estimates $\left[\hat{\mathbf{a}}_{k}^{T} \hat{\mathbf{b}}_{k}^{T}\right]^{T}, k=1,2, \ldots, L$ via any method \\
1 & Construct $\hat{\mathbf{x}}_{k}=\mathbf{x}-\sum_{i \neq k} \hat{\boldsymbol{\Phi}}_{i} \hat{\mathbf{a}}_{i} k=1,2, \ldots, L$ \\
2 & Maximize $\boldsymbol{\Lambda}_{k}=-N(\ln \pi+2 \ln \sigma)-\left(1 / \sigma^{2}\right)\left\|\hat{\mathbf{x}}_{k}-\boldsymbol{\Phi}_{k} \mathbf{a}_{k}\right\|^{2}, k=1,2, \ldots, L$ \\
3 & Update the initial estimates with maximization results in Step 2, and go to Step 1 \\
\hline
\end{tabular}

combination of all the other components together with the noise. But if we knew, somehow, the other components given by

$$
d_{k}[n]=\sum_{i \neq k} a_{i}[n] e^{j \varnothing_{i}[n]},
$$

then we would be able to define the following new data vector:

$$
x_{k}[n]=x[n]-d_{k}[n], \quad n=0,1, \ldots, N-1 .
$$

In that case the problem would be, given the data sequence

$$
x_{k}[n]=a_{k}[n] e^{j \varnothing_{k}[n]}+w[n], \quad n=0,1, \ldots, N-1,
$$

estimate the parameters $\mathbf{a}_{k}$ and $\mathbf{b}_{k}$. As we are going to estimate the phase and amplitude parameters of the $k$ th component, $x_{k}[n]$ can be considered as the complete data in the EM context. Similar to multicomponent case given in Section 2 the matrix notation and related logarithmic likelihood function for this single component case is

$$
\begin{aligned}
& \mathbf{x}_{k}=\boldsymbol{\Phi}_{k} \mathbf{a}_{k}+\mathbf{w}, \\
& \boldsymbol{\Lambda}_{k}=-N(\ln \pi+2 \ln \sigma)-\frac{1}{\sigma^{2}}\left\|\overline{\mathbf{x}}_{k}-\Psi_{k} \mathbf{a}_{k}\right\|^{2} .
\end{aligned}
$$

The minimization can be done either by PO method or by the proposed APA method in Section 3.

But, since we do not know the other components, we would not be able to compute the summation $d_{k}[n]$ given in (35). The only thing that we can do is to get an estimate for the other components. This is what the EM method suggests us. Therefore, for all components, the following EM iteration steps are carried out.

The EM iterations given in Table 5 will be carried out for sufficiently many times and when there is no significant change in the value of estimates compared to previous iteration, the iterations will be stopped.

The important thing in the EM method is that the initial estimates should be close enough to the actual values so that the estimate for complete data $\widehat{\mathbf{x}}_{k}$ given at Step 1 is not too deteriorated compared to its actual.

Actually the alternating phase and amplitude minimization proposed in Section 3 can also be considered as an application of EM method. While for the minimization of $f_{\mathbf{b}}(\mathbf{b})$ the amplitude parameters a are the missing or hidden variables, for the minimization of $f_{\mathbf{a}}(\mathbf{a})$ the phase parameters are missing or hidden variables.
During each EM iteration a monocomponent system of equation given by (38) is constructed. The related objective function is minimized by proposed APA method. Then this is done for all components and overall steps are repeated for a number of EM iterations. Since the order of computation cost for APA is $O\left(N N_{\mathbf{a}}\right)+O\left(N N_{\mathbf{b}}\right)$ and does not involve squares of $N_{\mathbf{a}}$ and $N_{\mathbf{b}}$, minimizing one by one is expected to have a comparable computational cost to that of multicomponent case. But since we repeat overall steps for a number of EM iterations, the cost will increase at a ratio of number of EM iterations. Also since during each EM step we need to compute $d_{k}[n]$ and $x_{k}[n]$ given by (35) and (36), this requires going from parameter space to component or signal space and will also increase computations. Therefore using EM with proposed APA method will increase the computational cost compared to APA method. But, it will be still less than the cost of phaseonly method, because, the phase-only method has $O\left(N N_{\mathrm{a}}^{2}\right)+$ $O\left(N N_{\mathbf{b}}\right)+O\left(N \sum_{i=1}^{L} \bar{P}_{i} \bar{Q}_{i}\right)$ order computation, while EM will approximately have $O\left(R_{\mathrm{EM}} N N_{\mathbf{a}}\right)+O\left(R_{\mathrm{EM}} N N_{\mathbf{b}}\right)$ order computations, where $R_{\mathrm{EM}}$ is the number of the EM iterations.

\section{Cramer-Rao Bounds for Mean Square Reconstruction Error}

Before comparing the proposed APA method with any other method in terms of computational cost, we first need to compare them in terms of attainable mean square reconstruction error performance. For that purpose we need to have the Cramer-Rao bounds on selected performance criteria.

Given the likelihood function $\Lambda$ in (14) the Fisher Information Matrix (FIM) for the parameter set $\boldsymbol{\theta}=\left[\mathbf{b}^{T} \mathbf{a}^{T}\right]^{T}$ is obtained by

$$
F_{i j}=-E\left\{\frac{\partial^{2} \Lambda}{\partial \theta_{i} \partial \theta_{j}}\right\} .
$$

The matrix is obtained [15] as

$$
\mathbf{F}=\frac{2}{\sigma^{2}} \operatorname{Re}\left\{\left[[\mathbf{A} \Phi]^{\mathbf{H}}[\mathbf{A} \boldsymbol{\Phi}]\right]\right\},
$$

where

$$
\begin{aligned}
& \mathbf{A}=\left[\begin{array}{lllll}
\mathbf{A}_{1} & \mathbf{A}_{2} & \mathbf{A}_{3} & \cdots & \mathbf{A}_{L}
\end{array}\right], \\
& \mathbf{A}_{i}=j\left[p_{0}[\mathbf{n}] \bullet s_{i}[\mathbf{n}] p_{1}[\mathbf{n}] \bullet s_{i}[\mathbf{n}] p_{2}[\mathbf{n}]\right. \\
& \left.\bullet[\mathbf{n}] \cdots p_{Q_{i}}[\mathbf{n}] \bullet s_{i}[\mathbf{n}]\right] \text {, }
\end{aligned}
$$


TABLE 6: Amplitude and phase orders for the components.

\begin{tabular}{|c|c|c|c|c|c|c|}
\hline \multirow{2}{*}{ Polynomial orders } & \multicolumn{2}{|c|}{ Component 1} & \multicolumn{2}{|c|}{ Component 2} & \multicolumn{2}{|c|}{ Component 3} \\
\hline & Amplitude & Phase & Amplitude & Phase & Amplitude & Phase \\
\hline Ex1 & 10 & 3 & 20 & 1 & & \\
\hline Ex2 & 10 & 3 & 10 & 3 & & \\
\hline Ex3 & 10 & 1 & 10 & 2 & 10 & 2 \\
\hline
\end{tabular}

where $s_{i}[\mathbf{n}]$ is the signal vector obtained by taking values at each time instant and "•" denotes component-bycomponent vector multiplication. An important property of the FIM for $\Lambda$ is that it does not depend on a and b directly but, rather, through phase functions $\varnothing_{i}[n]$ and signal components, $s_{i}[\mathbf{n}]$. It also depends on basis functions.

Cramer-Rao bound on variances (auto and cross) of the ML estimates of the parameter set $\boldsymbol{\theta}=\left[\mathbf{b}^{T} \mathbf{a}^{T}\right]^{T}$ is simply the inverse of FIM [22], that is,

$$
\operatorname{CRB}(\boldsymbol{\theta})=\mathbf{F}^{-1} .
$$

In an actual application rather than $\mathbf{a}$ and $\mathbf{b}$ parameters, we will be interested in signal components $s_{i}[n]$. Therefore, we will drive the bounds on the variance of the estimate for the signal components at time instant $n$. The component $s_{i}[n]$ is a function of the parameter set $\boldsymbol{\theta}_{i}=\left[\mathbf{b}_{i}^{T} \mathbf{a}_{i}^{T}\right]^{T}$. Having $\operatorname{CRB}\left(\boldsymbol{\theta}_{i}\right)$, which is a submatrix of $\operatorname{CRB}(\boldsymbol{\theta})$, the $\operatorname{CRB}\left(s_{i}[n]\right)$ can be obtained as [23]

$$
\mathrm{CRB}\left(s_{i}[n]\right)=\left(\mathbf{s}_{i, n}^{\prime}\right)^{H} \mathrm{CRB}\left(\boldsymbol{\theta}_{i}\right) \mathbf{s}_{i, n}^{\prime},
$$

where

$$
\mathbf{s}_{i, n}^{\prime}=\frac{\partial s_{i}[n]}{\partial \boldsymbol{\theta}_{i}}
$$

Using (4), and (51) $\mathbf{s}_{i}^{\prime}$ will be obtained as

$$
\mathbf{s}_{i, n}^{\prime}=\left[\begin{array}{ll}
\mathbf{A}_{i}[n] & \boldsymbol{\Phi}_{i}[n]
\end{array}\right]^{T} .
$$

$\mathbf{s}_{i, n}^{\prime}$ is simply the transpose of the row of $[\mathbf{A} \boldsymbol{\Phi}]$ corresponding to time instant $n$.

Since in our application we have $N$ time instants we need to compute (44) for all of them. But, in order to get an overall performance indication, we will sum them up and obtain the following bound as a reference for the component reconstruction error performance:

$$
\mathrm{CRB}\left(s_{i}\right)=\sum_{n=0}^{N-1} \mathrm{CRB}\left(s_{i}[n]\right),
$$

where $s_{i}$ denotes the $i$ th component. This is the total variance bound for the estimate of the signal values at all time instants between 0 and $N-1$.

\section{Simulation Results}

Though in terms of computation cost some comparison between proposed APA method and phase-only method is given in Section 4, in this section some simulation results are given. For the simulation, three nonstationary multicomponent signals were selected. The first two examples have two components, and the last example has three components. The real part of components and the magnitude STFT plot of the multicomponent signals are given in Figures 1 and 2.

All the examples were selected to be nonstationary signals with 256 samples. The components for the examples were obtained by sampling the following amplitude and phase functions selected with proper parameters and time shifting:

$$
\begin{aligned}
a(t, \alpha) & =\sqrt[4]{2 \alpha} e^{-\pi \alpha t^{2}}, \\
\phi\left(t, f_{c}, \beta, \gamma\right) & =\pi\left(2 f_{c} t+\beta t^{2}+\gamma t^{3}\right) .
\end{aligned}
$$

While Ex1 and Ex2 include components with quadratic phase terms, Ex3 includes two chirps and a Gaussian pulse. Since the phase terms are already polynomials, their orders were taken directly for the simulation. But since the amplitude parts are obtained by a Gaussian pulse, their polynomial fit orders were used. The polynomial orders for the examples are given in Table 6.

Simulation was carried out as follows: For a given noise realization, the initial estimates $\hat{\mathbf{a}}^{0}=\hat{\mathbf{a}}^{\mathrm{TF}}$ and $\hat{\mathbf{b}}^{0}=\hat{\mathbf{b}}^{\mathrm{TF}}$ were obtained from TFD. Then, using this initial phase parameters, $J(\mathbf{b})$ was minimized by iterating the BFGS algorithm up to some maximum number of steps. The maximum number of steps was set to values $4,6,8,10,14$, 20 , and 26 respectively and for each one the reconstruction error defined by

$$
e_{i}=\sum_{n=0}^{N-1}\left|\hat{s}_{i}[n]-s_{i}[n]\right|^{2},
$$

was computed for each component. This error, when averaged for many simulation runs, will give us, for a component, the total of experimental mean square reconstruction error for all time instants and will be compared to corresponding Cramer-Rao Bound given by (47).

Then proposed APA method was iterated with the same initial conditions used for minimization of $J(\mathbf{b})$ and with three different scenarios which defines number of the phase iterations and the alternating cycles. Then the minimization with PO and APA was repeated for another noise realization. 

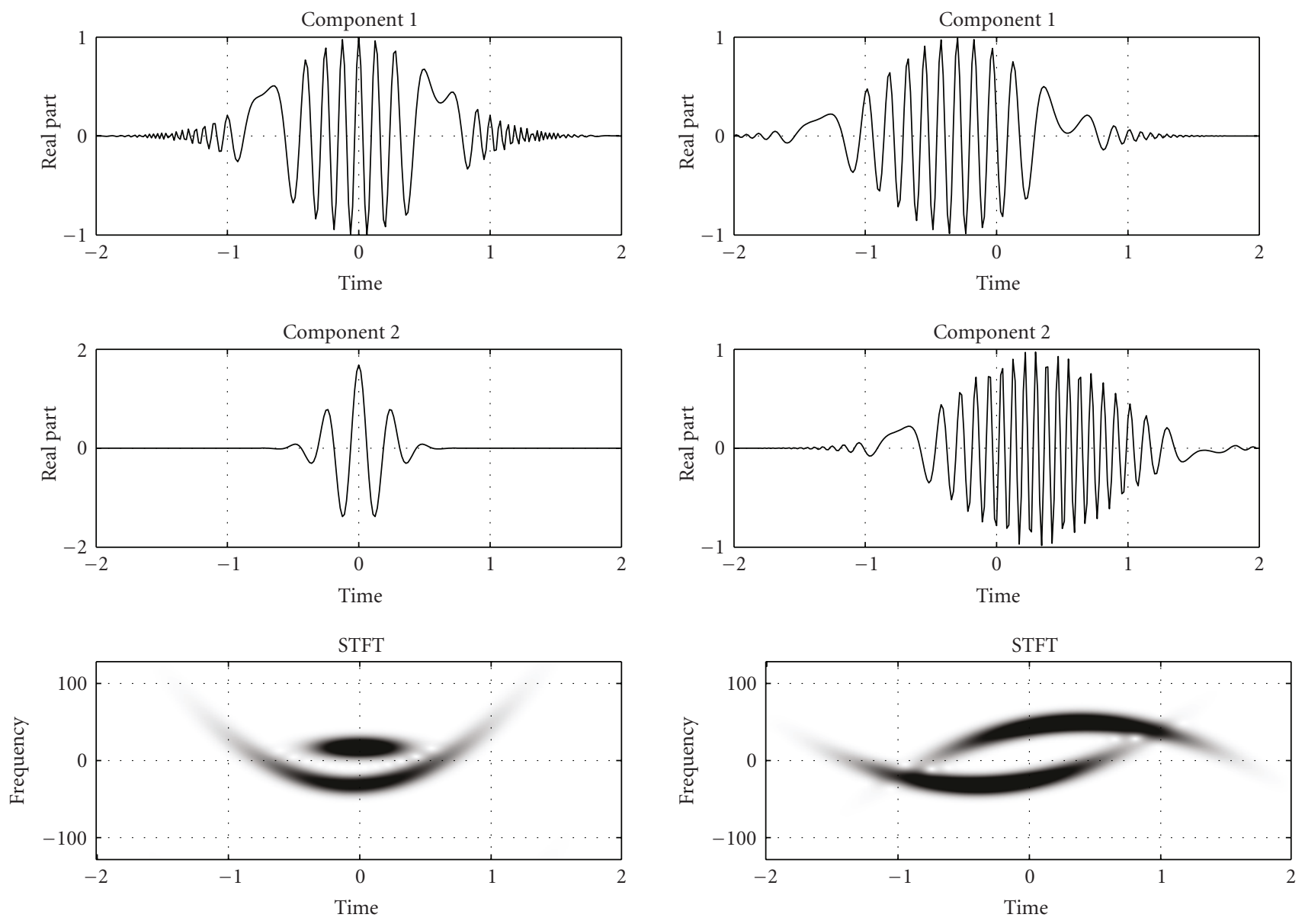

Figure 1: The Multicomponent signal examples Ex1 (left) and Ex2 with two components.

In first scenario of the APA method, denoted by APA1, the number of phase iterations for the minimization of $f_{\mathbf{b}}(\mathbf{b})$ was taken as the half of that used for minimization of $J(\mathbf{b})$. The number of alternating cycles for APA1 was selected as 5. For the second scenario, denoted by APA2, the phase iterations for the minimization of $f_{\mathbf{b}}(\mathbf{b})$ was taken the same as used for $J(\mathbf{b})$ and the number of alternating cycles was selected as 8 . The third scenario was the EM algorithm with the same conditions as APA1. The EM algorithm given in Table 5 was repeated for 4 iterations.

In all scenarios with proposed method, the amplitude parameters were computed analytically. Looking at Table 4 it is seen that, compared to minimization of $f_{\mathbf{b}}(\mathbf{b})$, the cost of minimization of $f_{\mathbf{a}}(\mathbf{a})$ is lower substantially, because the main contribution to computation cost of minimizing $f_{\mathbf{a}}(\mathbf{a})$ comes from initialization step and this step is computed once per alternating cycle. Similarly, if minimum $f_{\mathbf{a}}(\mathbf{a})$ is found analytically, the cost is again small compared to phase cost.

The quasi-Newton (BFGS) was implemented with line search algorithm suggested by Nocedal and Wright [24] which saves the gradient computations as much as possible. Therefore, the minimization of $J(\mathbf{b})$ is even favored.

Using the above scenarios for each SNR value between $8 \mathrm{~dB}$ and $20 \mathrm{~dB}$ the simulation was carried out for 400 runs.
During each run, together with component reconstruction error, the total number of function and gradient evaluations was also measured for each method and scenario. By averaging 400 runs the average of the reconstruction error given by (49) and average of the function and gradient evaluations were computed. Based on average function and gradient evaluations the computation cost for each method and scenario was obtained.

Using simulation results two groups of figures were obtained. In Figures 3, 4, 5, and 6 the attained average reconstruction error (MSE) versus SNR is plotted for proposed APA method and for the phase-only (PO) method given in [15]. On these figures the corresponding CramerRao Bound (CRB) computed by (47) is also plotted. PO stands for phase only method. APA1, APA2, and EM stand for proposed method with scenario 1, 2, and Expectation Maximization respectively.

On the other hand in Figures 7-12 the attained average reconstruction error versus required computation cost, in terms of millions of multiplications, is plotted for three SNR values. These are $8 \mathrm{~dB}, 14 \mathrm{~dB}$, and $20 \mathrm{~dB}$. In these figures also the Cramer Rao Bound (CRB) is shown as a bottom line.

In first group of figures the aim is to show that, for a given SNR value and the same initial conditions, the 

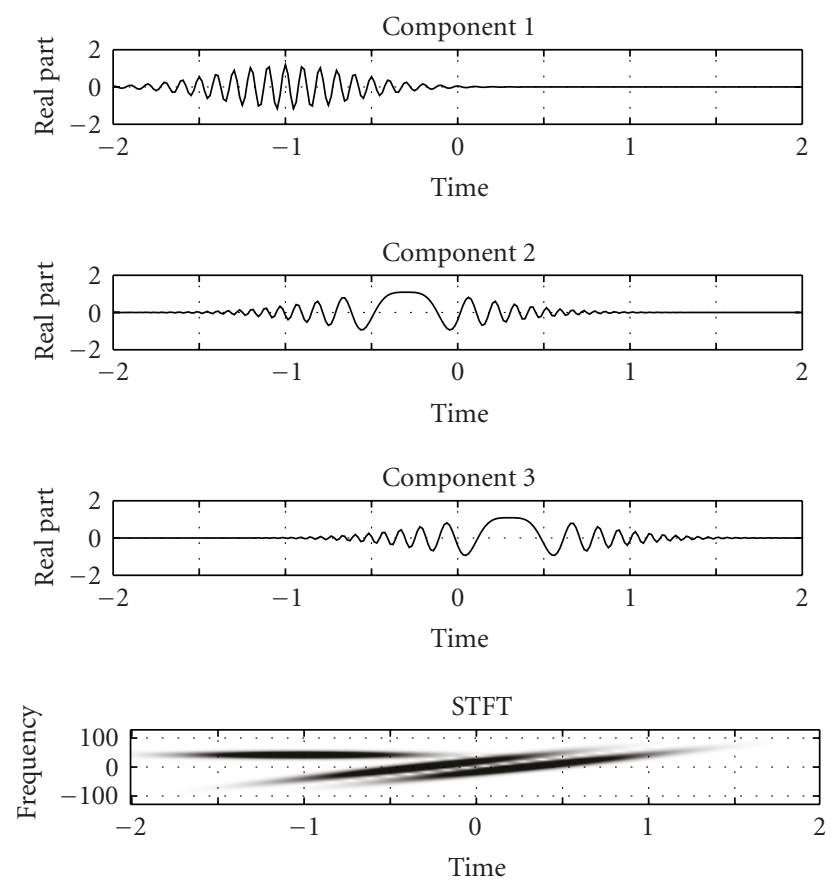

FIgUre 2: The Multicomponent signal example Ex3 with 3 components.

proposed method converges to comparable or even at some cases to better reconstruction error levels than phase-only method [15]. But in the second group of figures the aim is to show that, for a given SNR value and the same initial conditions, whatever the attained reconstruction error level, the proposed method converges with substantially less number of multiplications.

From Figures 3-6 we see that the proposed method with scenarios APA1, APA2, and EM has a comparable error performance to the phase-only method. While for Ex1 the performance of EM is better than the others, for other examples the performance is comparable. Therefore, with the proposed APA method and EM method that uses APA, we are able to solve the optimization problem in (15) iteratively and reach a comparable MSE performance compared to PO method. On the other hand the computational cost performance of the proposed APA and EM method is significantly better than that of PO method, that is, the proposed method saves the computations substantially.

From Figures 7-12 this situation can be observed clearly. For example, in Figure 7, which shows the average reconstruction error for component 1, with the proposed method using first scenario (APA1) the final reconstruction error level is reached by around 3 million multiplications. A similar level is reached with more than 20 million multiplications by PO method. The multiplication required for the same level for second scenario (APA2) is around 6 millions. On the other hand using EM a better error level is obtained. Similar results can be observed for component 2 as given in Figure 8 . From Figures 11 and 12 we see that again for Ex2 and Ex3 at SNR $8 \mathrm{~dB}$ the proposed method reaches final reconstruction error faster than PO method.

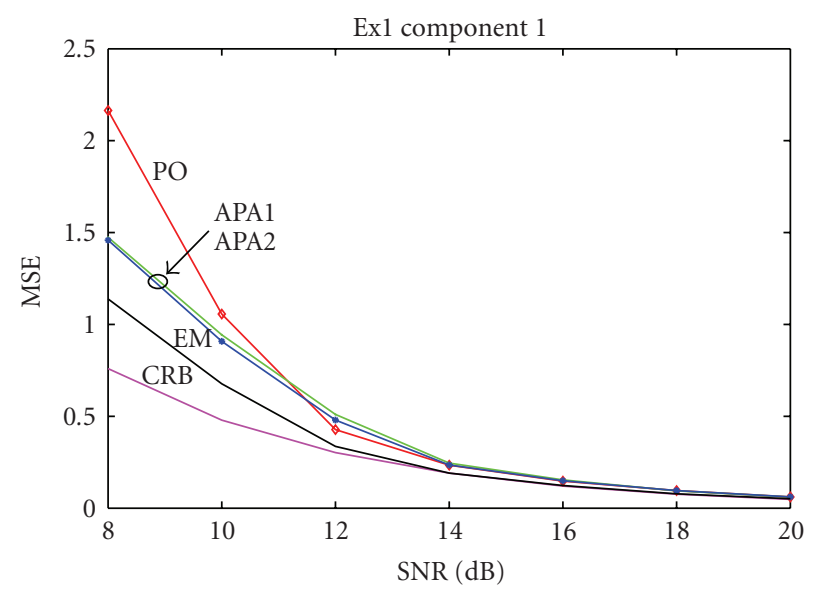

Figure 3: Experimental MSE versus SNR for Ex1 component 1.

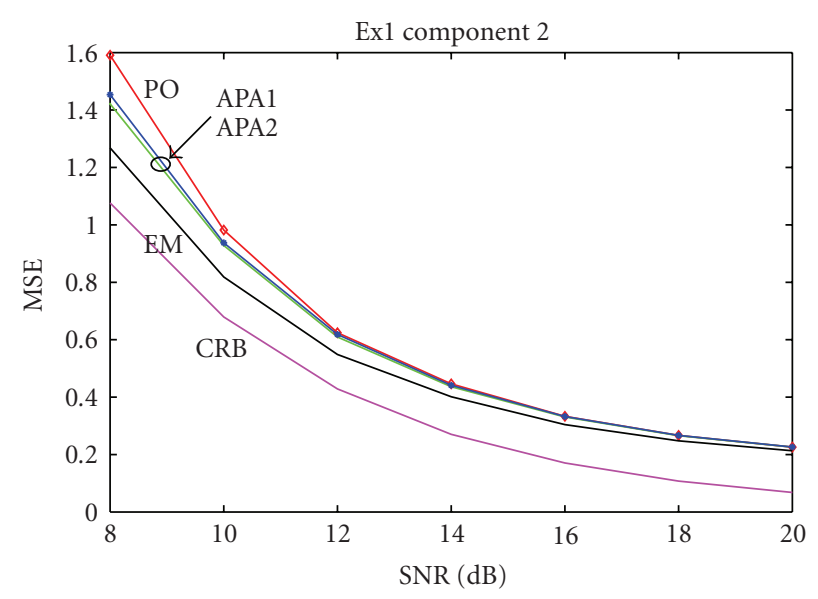

FIGURE 4: Experimental MSE versus SNR for Ex1 component 2.

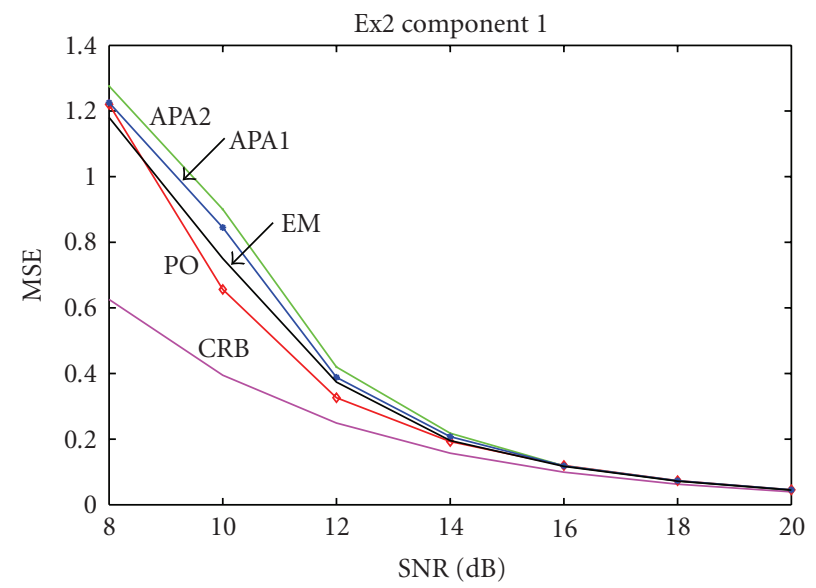

FIGURE 5: Experimental MSE versus SNR for Ex2 component 1.

As can be seen from Figures 9 and 10 Increasing SNR to 14 or $20 \mathrm{~dB}$ for Ex1 makes the benefit of using APA1 or APA2 apparent. The same advantage was observed for Ex2 and Ex3 also. While at low SNR EM is usually better than the others as the SNR increases, the advantage of EM is vanishing. 


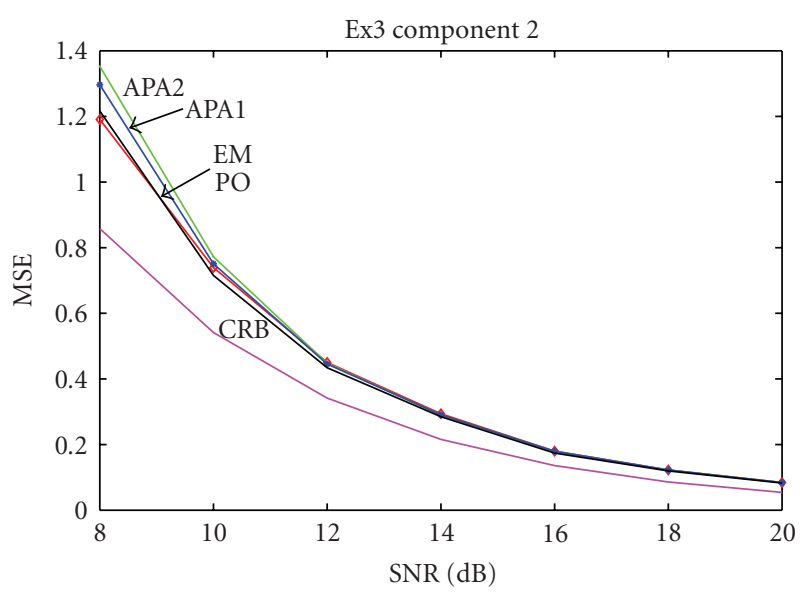

Figure 6: Experimental MSE versus SNR for Ex3 component 2.

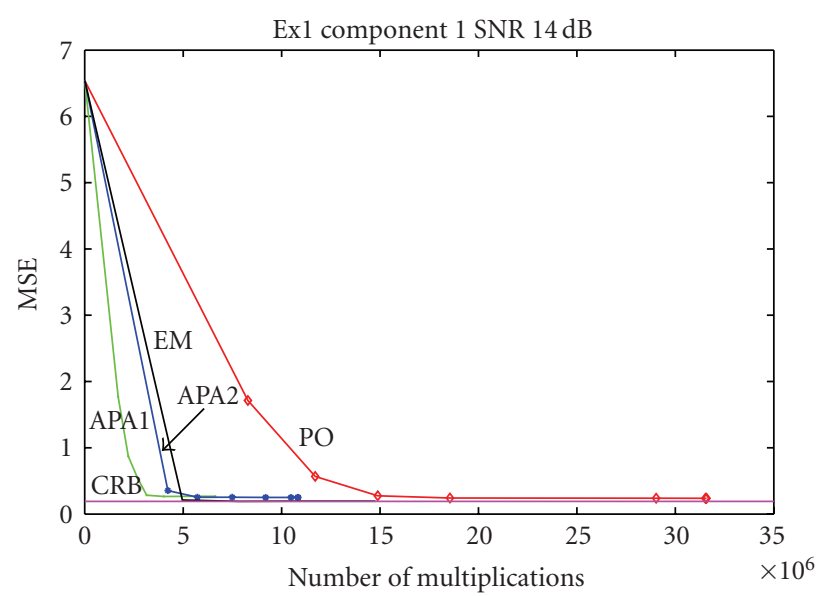

Figure 9: Experimental MSE versus computation cost for Ex1 at $14 \mathrm{~dB}$ (component 1).

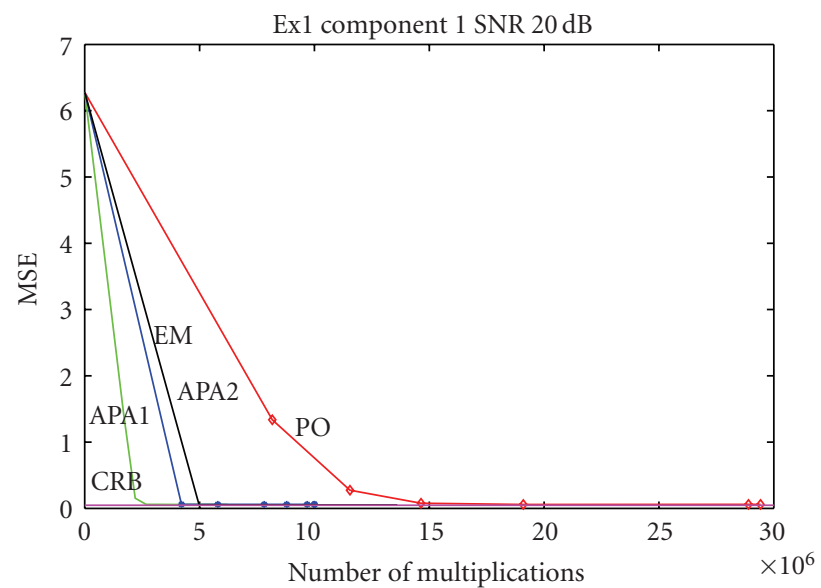

FIgure 10: Experimental MSE versus computation cost for Ex1 at $20 \mathrm{~dB}$ (component 1).

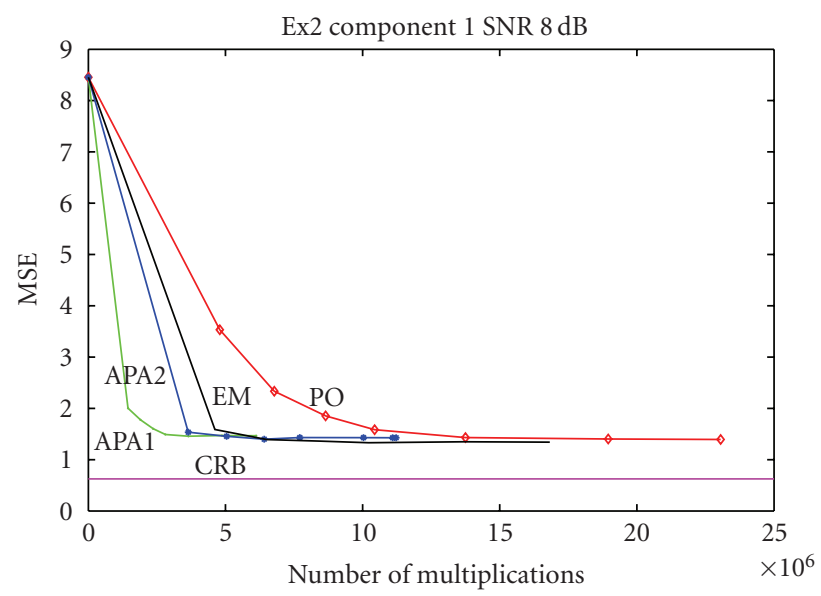

FIGURE 11: Experimental MSE versus computation cost for Ex2 at $8 \mathrm{~dB}$ (component 1).
Figure 8: Experime
$8 \mathrm{~dB}$ (component 2).

Ex1 component 2 SNR $8 \mathrm{~dB}$

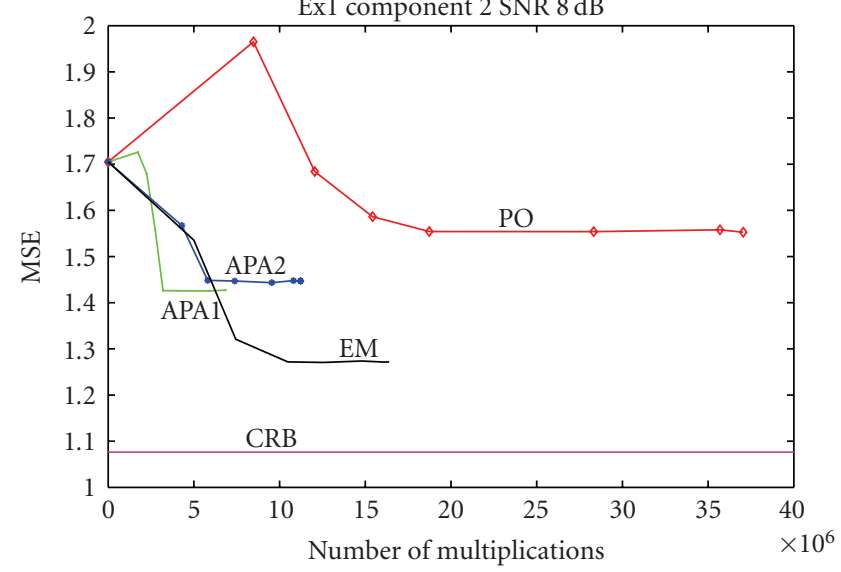

tal MSE versus computation cost for Exl at
Ex1 component 1 SNR $8 \mathrm{~dB}$

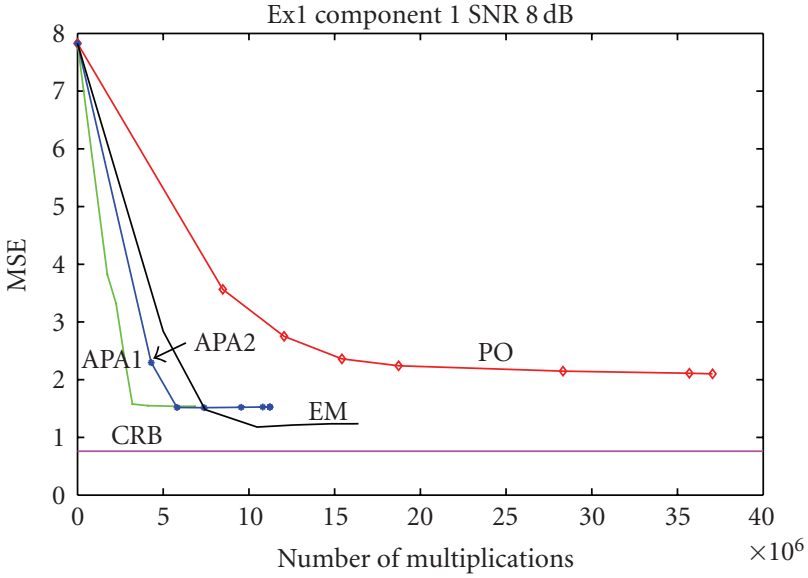

Figure 7: Experimental MSE versus computation cost for Ex1 at $8 \mathrm{~dB}$ (component 1). 


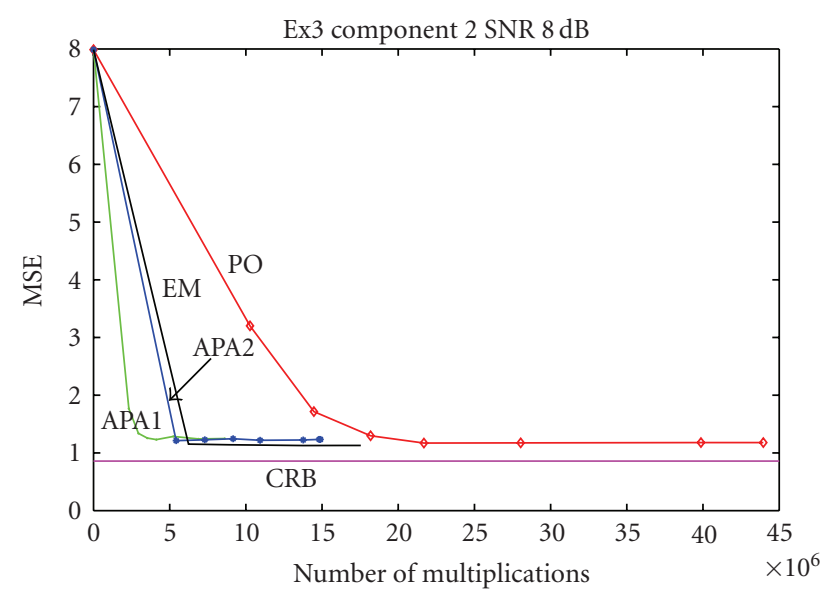

Figure 12: Experimental MSE versus computation cost for Ex3 at $8 \mathrm{~dB}$ (component 2).

Looking at all the selected examples for all SNR values it is clear that the proposed APA method has a superior performance in terms of computations required to reach a reconstruction error. APA1 which keeps alternating cycles and phase iterations lower than APA2 is superior at high SNR.

\section{Conclusion}

An iterative method has been proposed to estimate the components of a multicomponent signal via parametric ML estimation. The components on the TF plane are assumed to be well separated. Though can be estimated, it was also assumed that the number of components and polynomial orders for amplitude and phase functions are known. The resultant minimization problem was divided into separate amplitude and phase minimizations. With the proposed alternating phase and amplitude minimizations, the computation cost of original minimization problem reduced significantly. Also via simulations it was shown that, at low SNR, a better reconstruction error is achieved when the proposed method is used in an EM algorithm.

The initial estimates were obtained from time-frequency distribution. They can also be obtained via PPT. Depending on the performance of method by which initial estimates are obtained, good initial conditions can be obtained, and the computations can be saved even further.

\section{References}

[1] L. Cohen, "What is a multicomponent signal," in Proceedings of the International Conference on Acoustics, Speech, and Signal Processing, vol. 5, pp. 113-116, 1992.

[2] H. M. Ozaktaş, Z. Zalevsky, and M. A. Kutay, The Fractional Fourier Transform with Applications in Optics and Signal Processing, John Wiley \& Sons, New York, NY, USA, 2000.

[3] L. B. Almeida, "Fractional fourier transform and timefrequency representations," IEEE Transactions on Signal Processing, vol. 42, no. 11, pp. 3084-3091, 1994.
[4] H. M. Ozaktas, B. Barshan, D. Mendlovic, and L. Onural, "Convolution, filtering, and multiplexing in fractional Fourier domains and their relation to chirp and wavelet transforms," Journal of the Optical Society of America A, vol. 11, no. 2, pp. 547-559, 1994.

[5] G. F. Boudreaux-Bartels and T. W. Parks, "Time-varying filtering and signal estimation using Wigner distribution synthesis techniques," IEEE Transactions on Acoustics, Speech, and Signal Processing, vol. 34, no. 3, pp. 442-451, 1986.

[6] T. A. C. M. Claasen and W. F. G. Mecklenbraiiker, "The Wigner distribution-A tool for time-frequency signal analysis; Part 111: relations with other time-frequency signal transformations," Philips Journal of Research, vol. 35, no. 6, pp. 372-389, 1980.

[7] W. Krattenthaler and F. Hlawatsch, "Time-frequency design and processing of signals via smoothed Wigner distributions," IEEE Transactions on Signal Processing, vol. 41, no. 1, pp. 278287, 1993.

[8] G. C. Gaunaum and H. C. Strifors, "Signal analysis by means of time-frequency (Wigner-Type) distributions-applications to sonar and radar echoes," Proceedings of the IEEE, vol. 84, no. 9, pp. 1231-1248, 1996.

[9] K. B. Yu and S. Cheng, "Signal synthesis from Pseudo-Wigner distribution and applications," IEEE Transactions on Acoustics, Speech, and Signal Processing, vol. 35, no. 9, pp. 1289-1302, 1987.

[10] L. Cohen, “Time-frequency distributions - a review," Proceedings of the IEEE, vol. 77, no. 7, pp. 941-981, 1989.

[11] D. L. Jones and R. G. Baraniuk, "Adaptive optimal-kernel time-frequency representation," IEEE Transactions on Signal Processing, vol. 43, no. 10, pp. 2361-2371, 1995.

[12] L. Cohen and T. E. Posch, "Positive time-frequency functions," IEEE Transactions on Acoustics, Speech, and Signal Processing, vol. 33, no. 1, pp. 31-38, 1985.

[13] P. J. Loughlin, J. W. Pitton, and L. E. Atlas, "Construction of positive time-frequency distributions," IEEE Transactions on Signal Processing, vol. 42, no. 10, pp. 2697-2705, 1994.

[14] B. Friedlander, "Parametric signal analysis using the polynomial phase transform," in Proceedings of the IEEE Signal Processing Workshop Higher Order Statistics, Stanford Sierra Camp, South Lake Tahoe, Calif, USA, June 1993.

[15] B. Friedlander and J. M. Francos, "Estimation of amplitude and phase parameters of multicomponent signals," IEEE Transactions on Signal Processing, vol. 43, no. 4, pp. 917-926, 1995.

[16] S. Peleg, Estimation and detection with the discrete polynomial transform, Ph.D. dissertation, Department of Electrical and Computer Engineering, University of California, Davis, Calif, USA, 1993.

[17] D. S. Pham and A. M. Zoubir, "Analysis of multicomponent polynomial phase signals," IEEE Transactions on Signal Processing, vol. 55, no. 1, pp. 56-65, 2007.

[18] A. Francos and M. Porat, "Analysis and synthesis of multicomponent signals using positive time-frequency distributions," IEEE Transactions on Signal Processing, vol. 47, no. 2, pp. 493504, 1999.

[19] D. B. Luenberger and Y. Ye, Linear and Nonlinear Optimization, Springer, New York, NY, USA, 3rd edition, 2008.

[20] A. P. Dempster, N. M. Laird, and D. B. Rubin, "Maximum likelihood from in-complete data via the em algorithm," Journal of the Royal Statistical Society, vol. 39, no. 1, pp. 1-38, 1977. 
[21] G. McLachlan and T. Krishnan, The EM Algorithm and Extensions, John Wiley \& Sons, New York, NY, USA, 1996.

[22] S. Zacks, The Theory of Statistical Inference, John Wiley \& Sons, New York, NY, USA, 1971.

[23] C. R. Rao, Linear Statistical Inference and Its Applications, John Wiley \& Sons, New York, NY, USA, 1965.

[24] J. Nocedal and S. J. Wright, Numerical Optimization, Springer, New York, NY, USA, 1999. 